\title{
Difference in Local Lung Movement During Tidal Breathing Between COPD Patients and Asthma Patients Assessed by Four-dimensional Dynamic-ventilation CT Scan
}

This article was published in the following Dove Press journal:

International Journal of Chronic Obstructive Pulmonary Disease

\author{
Eisuke Mochizuki' \\ Yoshiihiro Kawai $^{2}$ \\ Keisuke Morikawa' \\ Yutaro Ito' \\ Namio Kagoo' \\ Tsutomu Kubota' \\ Koshiro Ichijyo' \\ Masahiro Uehara' \\ Masanori Harada' \\ Shun Matsuura' \\ Masaru Tsukui' \\ Naoki Koshimizu' \\ 'Department of Respiratory Medicine, \\ Fujieda Municipal Hospital, Fujieda 426- \\ 8677, Japan; ${ }^{2}$ Department of Radiology, \\ Fujieda Municipal Hospital, Fujieda 426- \\ 8677, Japan
}

Correspondence: Eisuke Mochizuki

Tel +8I-54-646-IIII

$\mathrm{Fax}+81-54-646-1 / 22$

Email eisukefukui@gmail.com
Background: The validity of four-dimensional dynamic-ventilation CT scan for distinguishing COPD from asthma has not been established.

Purpose: To assess whether four-dimensional dynamic-ventilation CT scan can aid in the diagnosis of COPD by comparing local lung movement during tidal breathing between COPD and asthma.

Patients and Methods: Thirty-three COPD patients (30 males and three females; median age 74; range 44-89 years) and 11 asthma patients (five males and six females; median age 55; range: 32-75 years) underwent whole-lung dynamic-ventilation CT scan. CT data were reconstructed, one respiratory cycle to 10 phases, and in addtion we reconstructed threefold new phase data sets. We then analyzed local lung movement during tidal breathing using unpaired $t$-tests and chi-squared tests.

Results: The local lung movement in COPD patients was significantly smaller than in asthma patients, especially in the ventral part of the lung. This was so even in patients who had mild emphysema (Goddard score $<8$ ).

Conclusion: Quantitative evaluation using four-dimensional dynamic-ventilation CT scan demonstrated that local lung movement during tidal breathing, particularly in the ventral lung, was smaller in COPD than in asthma patients, which may help distinguish COPD from asthma.

Keywords: COPD, asthma four-dimensional dynamic-ventilation CT

\section{Introduction}

COPD is one of the leading causes of morbidity and mortality in the world and is sometimes difficult to distinguish from asthma. ${ }^{1}$ There are several available modalities to distinguish these disorders, including spirometry, sputum eosinophils, exhaled nitric oxide and forced oscillation technique. However, more useful techniques are needed, which are easy to perform and interpret.

Functional diaphragm analysis has been performed using many imaging techniques, including conventional chest radiography, fluoroscopy, ultrasonography, computed tomography, magnetic resonance imaging and dynamic chest phrenicography. $^{2-4}$

In addition, multidetector CT (MDCT) scanners can continuously scan the thorax during free breathing to visualize lung and diaphragm motion, which can 
be analyzed quantitatively. With this four-dimensional dynamic-ventilation CT scan, we can compare lung movement between diseases. ${ }^{5}$ However, the four-dimensional dynamic-ventilation CT scan has not been fully tested for whole-lung evaluation of local lung movement during breathing in COPD patients. The purpose of our study was therefore to quantitatively compare local lung movement during tidal breathing, between COPD and asthma patients, and clarify features of local lung movement between the two.

\section{Methodology}

\section{Patients}

This retrospective study was conducted at Fujieda Municipal Hospital in Japan and approved by the local ethics committee. All participants provided written informed consent. From June 2018 to February 2020, nonconsecutive COPD $(n=33)$ and asthma $(n=11)$ patients who met the following inclusion criteria were recruited: (1) COPD diagnosed according to the Global Initiative for Chronic Obstructive Lung Disease criteria ${ }^{6}$ without acute respiratory infection or other respiratory diseases such as bronchiectasis or interstitial lung disease; (2) current or exsmokers with more than 10 pack-years; (3) over 40 years old; (4) scheduled for conventional chest radiography; (5) ability to follow instructions for tidal breathing. A total of 33 COPD patients (30 males, three females; median age, 74 years; age range: 44-89 years) were included in the analysis. We also recruited patients older than 18 years with bronchial asthma, according to the Global Initiative for Asthma (GINA), ${ }^{7}$ who attended our outpatient department. A total of 11 asthma patients (five males, six females; median age, 55 years; age range: 32-75 years) were included in the analysis.

All patients were well controlled, without exacerbations within the three months before spirometry and no patient with asthma was classified as severe. Patients with asthma COPD overlap were excluded from this study.

\section{CT Scan}

All patients were scanned on a 16-row MDCT scanner, Aquilion LB (Canon Medical Systems, Otawara, Tochigi, Japan) for dynamic ventilation scans and 320-row MDCT scanner, Aquilion ONE (Canon Medical Systems) for conventional studies.

Patients underwent free breathing with respiratory gating signals acquired using the Respiratory Gating System,
AZ-733V (Anzai Medical, Tokyo, Japan) for dynamic ventilator scans. No contrast medium was used.

Patients were previously instructed on how to perform the respiratory maneuvers while lying on the CT scanner acquisition bed. Radiologic technologists confirmed that breathing was performed properly during the scan. The 4DCT scanned in helical mode using $2 \mathrm{~mm} \times 16$-row and beam pitch=0.063-0.119 (depending on the respiratory cycle), was reconstructed into ten respiratory phase bins with $0.78 \times 0.78 \times 2 \mathrm{~mm}$ voxels. Scanning field of view (FOV) was $400 \mathrm{~mm}$. Other scanning and reconstruction parameters for the dynamic ventilation CT were as follows: tube currents $=50 \mathrm{~mA}$; tube voltage $=120 \mathrm{kVp}$; rotation time $=0.5$ second; total scanning time $=54-92$ seconds; imaging FOV: $400 \mathrm{~mm}$; collimation $=2 \mathrm{~mm}$; slice thickness $=2 \mathrm{~mm}$; reconstruction image interval $=2 \mathrm{~mm}$; reconstruction kernel=FC52 (for lung field); reconstruction respiratory phase $b i n=10$; reconstruction method=half; iterative reconstruction method=Adaptive Iterative Dose Reduction using Threedimensional Processing (AIDR3D), weak setting.

Conventional static chest CT was also performed using helical scanning with $0.5 \mathrm{~mm} \times 80$-row. The parameters for the conventional chest $\mathrm{CT}$ were as follows: tube currents=automatic exposure control (AEC); tube voltage $=20$ $\mathrm{kVp}$; scanning method $=$ helical scanning; rotation time $=0.5$ second; beam pitch $=0.813$; imaging FOV: $320 \mathrm{~mm}$; collimation $=0.5 \mathrm{~mm} \times 80$-row; slice thickness $=2 \mathrm{~mm}$; reconstruction kernel=FC52 (for lung field); iterative reconstruction $=\mathrm{AIDR} 3 \mathrm{D}$ (weak setting). The $\mathrm{CT}$ dose index volume (CTDIvol) for the dynamic ventilation CT was 19.3-36.7 mGy. The dose-length product value (DLP) was 786-1306 $\mathrm{mGy} \mathrm{cm}$. The total estimated radiation exposure for the dynamic ventilation CT was $11.2-18.3 \mathrm{mSv}$.

\section{Image Reconstruction and Analysis of Local Lung Movement (Dynamic-Ventilation CT)}

Using a commercial software program (PhyZiodynamics, Ziosoft Inc., Minato-ku, Tokyo, Japan), we reconstructed the dynamic-ventilation CT data and evaluated the local lung movement.

Phyziodynamics uses motion coherent technique, performing nonlinear interpolation between registered voxels to generate up to fivefold phase data sets from the original phase exam. As it is possible to quantitatively evaluate displacement and time target voxels takes to move between 
previous and initial phase, we calculated "displacement" and "velocity", and we used phyziodynamics to reconstruct theefold new phase data sets from the original phase exam.

The software tracked a single designated center point of a three-dimensional spherical volume of interest (VOI) with a fixed volume $(1.5 \mathrm{cc})$ throughout the dynamic-ventilation CT. Then we tracked the titer of VOI in the respiratory phase starting from the peak inspiratory phase. We measured the "displacement" and "velocity" calculated by the software and evaluated the mean maximum "displacement", and mean expiratory and inspiratory phase "velocity". "displacement" was defined as the amount of target voxel movement relative to the initial phase. "velocity" was defined as target voxel velocity as it moved from the previous phase. With change of titer, we quantified local lung movement.

Measurement points were as follows: apex, CPA (costophrenic angle), top of diaphragm, ventral part of diaphragm, midpoint between apex and CPA and midpoint between ventral part of diaphragm and apex in both lungs, on sagittal plane at the level of the tracheal bifurcation (Figure 1).

\section{Image Analysis-emphysema Measurement on Chest CT}

We performed the measurement of emphysema on conventional chest CT using software Lung Checker version 1.5.0.0j (JMAC system, Sapporo, Japan). The percentage of low attenuation volume (LAV\%, <-950HU) was obtained automatically and then the Goddard score was calculated.

\section{Spirometry}

Within three months of the dynamic-ventilation CT date, all patients underwent spirometry. Measurements included
$\mathrm{FEV}_{1}$ ) and FVC, according to the American Thoracic Society standards. ${ }^{7}$

\section{Statistical Analysis}

Descriptive statistics are expressed as median with range for continuous variables and as frequency and percentages for nominal variables. Patients demographic characteristics and four-dimensional dynamic-ventilation CT scan parameters of diaphragm motion, were compared between COPD and asthma patients using Student's $t$-tests for continuous variables, and chi-squared for nominal variables. A $p$-value of $<0.05$ was considered significant and all tests were two-sided. All statistical analyses were performed with EZR (Saitama Medical Center, Jichi Medical University, Saitama, Japan), which is a graphical user interface for $R$ (The $\mathrm{R}$ Foundation for Statistical Computing, Vienna, Austria). More precisely, it is a modified version of $\mathrm{R}$ commander designed to add statistical functions frequently used in biostatistics). ${ }^{8}$

\section{Results \\ Patient Characteristics}

Table 1 summarizes the characteristics of the enrolled patients. Data in parentheses are the range. We found significant differences in age $(p<0.01)$, gender $(p<0.01)$, smoking index $(p<0.01)$, smoking status $(p<0.01)$, Goddard score $(p=0.03), \mathrm{FEV}_{1} \quad(p=0.03), \quad \mathrm{FEV}_{1} / \mathrm{FVC}$ $(p<0.01)$ and LAV\% $(p<0.01)$.

\section{Displacement and Velocity}

The results of displacement of each point is summarized in Table 2. The titer of the ventral point was significantly

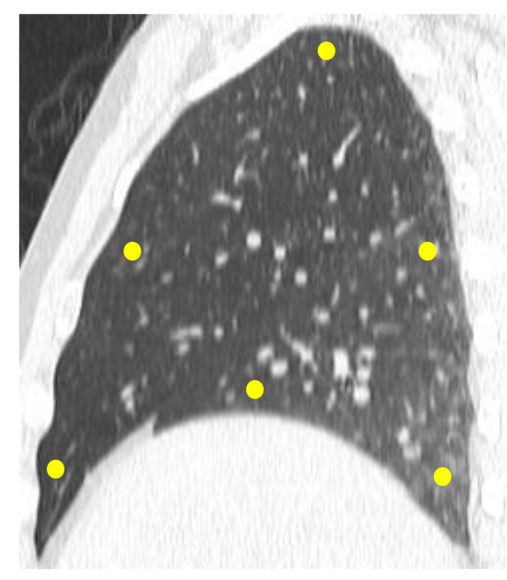

- Apex

- CPA

- Top of the diaphragm

- Ventral part

- Midpoint between apex and CPA

- Midpoint between ventral part and apex

Figure I The measurement point of lung movement in our study. 
Table I Characteristics of COPD Patients and Patients with Asthma

\begin{tabular}{|c|c|c|c|}
\hline & COPD $(n=33)$ & Asthma $(n=I I)$ & $p$-value \\
\hline Age (years) & $74(44-89)$ & $55(32-75)$ & $<0.01$ \\
\hline Gender (male/female) & $30 / 3$ & $5 / 6$ & $<0.01$ \\
\hline BMI $\left(\mathrm{kg} / \mathrm{m}^{2}\right)$ & $21.1(15.6-31.3)$ & $22.6(15.6-27.9)$ & 0.43 \\
\hline Alb $(g / d L)$ & $4.1(2.7-4.6)$ & $4.3(3.7-4.7)$ & 0.09 \\
\hline Smoking index (pack-years) & $43(0-150)$ & $0(0-5 I)$ & $<0.01$ \\
\hline Smoking status (current/ex/never) & $(5 / 27 / 1)$ & $(2 / 2 / 7)$ & $<0.01$ \\
\hline Goddard score & $6(0-12)$ & $6(5-6)$ & 0.03 \\
\hline GOLD $(\mathrm{I} / \mathrm{II} / \mathrm{III} / \mathrm{IV})$ & $12 / 12 / 9 / 0$ & $0 / 1 / 0 / 0^{a}$ & \\
\hline $\mathrm{FEV}_{1}(\mathrm{~L})$ & $1.43(0.7 \mathrm{I}-3.28)$ & $2.24(1.43-4.91)$ & 0.03 \\
\hline $\mathrm{FEV}_{\mathrm{l}} / \mathrm{FVC}(\%)$ & $55.9(29.7-68.9)$ & $73.2(55.0-79.4)$ & $<0.01$ \\
\hline TV (L) & $0.91(0.31-2.27)$ & $0.97(0.69-1.25)$ & 0.87 \\
\hline LAV\% (\%) & $13.7(0.4-33.9)$ & $0.9(0.3-19.8)$ & 0.01 \\
\hline
\end{tabular}

Notes: ${ }^{a}$ Eight did not have obstructive impairment. Two did not perform spirometry. Data presented as median and IQR or categorical variables were presented as numbers (n) and percentages (\%).

Table 2 Displacement of COPD Patients and Patients with Asthma

\begin{tabular}{|c|c|c|c|}
\hline & COPD $(n=33)$ & Asthma $(n=I I)$ & $p$-value \\
\hline \multicolumn{4}{|l|}{ Right } \\
\hline Apex & $1.9(0.7-4.1)$ & $3.1(0.6-6.0)$ & 0.06 \\
\hline CPA & $20.2(6.5-37.8)$ & $21.0(13.5-32.5)$ & 0.41 \\
\hline Top of the diaphragm & $13.3(6.9-27.1)$ & $15.5(\mid 1.2-29.5)$ & 0.04 \\
\hline Ventral part & $5.5(1.5-13.4)$ & $10.7(4.6-25.2)$ & $<0.01$ \\
\hline Midpoint between apex and CPA & $9.2(2.2-27.3)$ & $11.9(3.8-15.3)$ & 0.36 \\
\hline Midpoint between ventral part and apex & $4.5(1.5-9.0)$ & $7.4(2.5-17.1)$ & 0.13 \\
\hline \multicolumn{4}{|l|}{ Left } \\
\hline Apex & $1.89(0.7-4.1)$ & $3.41(0.9-6.8)$ & 0.23 \\
\hline CPA & $20.2(6.5-37.8)$ & $19.9(12.3-34.3)$ & 0.54 \\
\hline Top of the diaphragm & $13.3(6.9-27.1)$ & $15.4(10.7-33.9)$ & 0.19 \\
\hline Ventral part & $5.5(1.5-13.4)$ & I $3.3(4.8-20.8)$ & $<0.01$ \\
\hline Midpoint between apex and CPA & $9.2(2.2-27.3)$ & $14.8(5.4-16.3)$ & 0.03 \\
\hline Midpoint between ventral part and apex & $5.18(1.5-10.7)$ & $7.1(3.7-16.1)$ & 0.07 \\
\hline
\end{tabular}

Note: Data presented as median and IQR.

smaller in COPD patients than in asthma patients. This result was observed for both sides of the lung. At the top of the diaphragm of the right lung and midpoint between apex and CPA of the left lung, the titer was also significantly smaller in COPD patients. Tables 3 and 4 summarize the results of velocity-during inhalation and exhalation respectively. The titer of the ventral point was also smaller in COPD patients than in asthma patients, for both sides of the lung. The titer of top of the diaphragm for right side of the lung was also smaller in COPD patients.

\section{COPD Patients with Mild Emphysema}

We defined patients with Goddard score $<8$, as patients with mild emphysema. Table 5 summarizes the demographic feature of the COPD patients with mild emphysema and asthma. We confirmed significant differences in 
Table 3 Velocity During Inhalation of COPD Patients and Patients with Asthma

\begin{tabular}{|c|c|c|c|}
\hline & COPD $(n=33)$ & Asthma $(n=I I)$ & p-value \\
\hline \multicolumn{4}{|l|}{ Right } \\
\hline Apex & $6.8(0.4-16.7)$ & $10.7(1.6-24.7)$ & 0.06 \\
\hline CPA & $64.4(32.4-147.7)$ & $79.9(48.7-116.3)$ & 0.35 \\
\hline Top of the diaphragm & $45.8(20.9-90.4)$ & $55.9(35.6-105.0)$ & 0.03 \\
\hline Ventral part & $21.1(6.8-46.6)$ & $48.7(19.0-74.4)$ & $<0.01$ \\
\hline Midpoint between apex and CPA & $34.5(8.8-74.5)$ & $35.6(11.9-70.5)$ & 0.61 \\
\hline Midpoint between ventral part and apex & $17.7(5.1-40.9)$ & $29.2(10.4-46.3)$ & 0.07 \\
\hline \multicolumn{4}{|l|}{ Left } \\
\hline Apex & $9.9(3.0-18.6)$ & $12.0(2.5-20.5)$ & 0.42 \\
\hline CPA & $64.9(31.8-143.1)$ & $70.9(33.0-97.5)$ & 0.31 \\
\hline Top of the diaphragm & $45.9(27.8-86.0)$ & $61.7(34.9-101.0)$ & 0.24 \\
\hline Ventral part & $17.7(8.8-55.9)$ & $47.7(20.2-69.2)$ & $<0.01$ \\
\hline Midpoint between apex and CPA & $32.2(13.2-58.9)$ & $42.9(15.2-56.5)$ & 0.16 \\
\hline Midpoint between ventral part and apex & I7.I (5.4-45.5) & $31.8(13.0-53.0)$ & 0.04 \\
\hline
\end{tabular}

Note: Data presented as median and IQR.

Table 4 Velocity During Exhalation of COPD Patients and Patients with Asthma

\begin{tabular}{|c|c|c|c|}
\hline & COPD $(n=33)$ & Asthma $(n=I I)$ & $p$-value \\
\hline \multicolumn{4}{|l|}{ Right } \\
\hline Apex & $6.2(1.8-16.2)$ & $8.2(2.6-21.8)$ & 0.35 \\
\hline CPA & $82.7(35.3-|4| .0)$ & $83.6(44.1-123.6)$ & 0.75 \\
\hline Top of the diaphragm & $46.2(22.2-91.5)$ & $66.2(36.2-89.7)$ & 0.03 \\
\hline Ventral part & I5.9 (5.6-50.9) & $4 I .1(11.3-80.2)$ & $<0.01$ \\
\hline Midpoint between apex and CPA & $32.4(6.9-67.4)$ & $38.4(14.5-78.8)$ & 0.71 \\
\hline Midpoint between ventral part and apex & I7.6 (5.2-39.3) & $24.0(7.5-60.8)$ & 0.20 \\
\hline \multicolumn{4}{|l|}{ Left } \\
\hline Apex & $7.8(3.5-16.7)$ & $10.2(3.2-2 \mid .4)$ & 0.96 \\
\hline CPA & $78.8(33.7-124.3)$ & $62.4(36.5-99.7)$ & 0.32 \\
\hline Top of the diaphragm & $51.1(22.2-85.8)$ & $49.8(32.1-95.5)$ & 0.81 \\
\hline Ventral part & $17.2(7.0-34.3)$ & $40.0(13.5-67.2)$ & $<0.01$ \\
\hline Midpoint between apex and CPA & $33.8($ (II.I-63.4) & $4 I . I(19.1-57.0)$ & 0.18 \\
\hline Midpoint between ventral part and apex & $15.8(6.9-33.7)$ & $18.5(13.2-47.5)$ & 0.10 \\
\hline
\end{tabular}

Note: Data presented as median and IQR.

age, gender, smoking index, smoking status and $\mathrm{FEV} / \mathrm{FVC}$.

\section{Displacement and Velocity (-Inhalation/ Exhalation) in COPD Patients with Mild Emphysema}

The titer of the ventral point was also smaller in COPD patients with mild emphysema than in asthma patients, for both sides of the lung, similar to results seen for all COPD patients, as shown in Tables 6-,8. At the top of the diaphragm of the right lung, the titer of displacement and velocity (-inhalation/exhalation) was also significantly smaller in COPD patients.

\section{Displacement and Velocity of Ventral Lung Over One Respiratory Cycle}

(Figures 2,,,) show the displacement and velocity of the ventral point of both lungs.

They demonstrate greater alteration for both displacement and velocity in asthma patients, compared with COPD patients. We further found that local lung movement during tidal breathing was significantly smaller in COPD patients 
Table 5 Characteristics of COPD Patients with Mild Emphysema and Patients with Asthma

\begin{tabular}{|c|c|c|c|}
\hline & COPD with Mild Emphysema $(n=20)$ & Asthma $(n=I I)$ & $p$-value \\
\hline Age (years) & $74(44-89)$ & $55(32-75)$ & $<0.01$ \\
\hline Gender (male/female) & $17 / 3$ & $5 / 6$ & 0.03 \\
\hline BMI $\left(\mathrm{kg} / \mathrm{m}^{2}\right)$ & $22.1(15.6-31.3)$ & $22.6(15.6-27.9)$ & 0.93 \\
\hline Alb $(g / d L)$ & $4.2(3.0-4.6)$ & $4.3(3.7-4.7)$ & 0.24 \\
\hline Smoking index (pack-years) & $41.8(0-88)$ & $0(0-5 I)$ & $<0.01$ \\
\hline Smoking status (current/ex/never) & $(5 / 14 / 1)$ & $(2 / 2 / 7)$ & $<0.01$ \\
\hline Goddard score & $6(0-7)$ & $6(5-6)$ & 0.91 \\
\hline GOLD (I/II/III/IV) & $8 / 10 / 2 / 0$ & $0 / 1 / 0 / 0^{a}$ & \\
\hline $\mathrm{FEV}_{1}(\mathrm{~L})$ & $1.79(0.78-3.28)$ & $2.24(1.43-4.91)$ & 0.11 \\
\hline $\mathrm{FEV}_{1} / \mathrm{FVC}(\%)$ & $61.3(39.6-68.9)$ & $73.2(55.0-79.4)$ & $<0.01$ \\
\hline TV (L) & $0.78(0.3 \mathrm{I}-2.27)$ & $0.97(0.69-1.25)$ & 0.21 \\
\hline LAV\% (\%) & $5.4(0.4-18.4)$ & $0.9(0.3-19.8)$ & 0.28 \\
\hline
\end{tabular}

Notes: ${ }^{a}$ Eight did not have obstructive impairment. Two did not perform spirometry. Data presented as median and IQR or categorical variables were presented as numbers (n) and percentages (\%).

Table 6 Displacement of COPD Patients with Mild Emphysema and Patients with Asthma

\begin{tabular}{|c|c|c|c|}
\hline & COPD with Mild Emphysema $(n=20)$ & Asthma $(n=I I)$ & $p$-value \\
\hline \multicolumn{4}{|l|}{ Right } \\
\hline Apex & $2.17(0.7-2.7)$ & $3.1(0.6-6.0)$ & 0.11 \\
\hline CPA & $18.5(10.2-32.4)$ & $21.0(13.5-32.5)$ & 0.16 \\
\hline Top of the diaphragm & I2.7 (7.4-24.4) & $15.5(\mid 1.2-29.5)$ & 0.01 \\
\hline Ventral part & $5.5(1.5-13.4)$ & $10.7(4.6-25.2)$ & $<0.01$ \\
\hline Midpoint between apex and CPA & $8.7(2.2-18.8)$ & $11.9(3.8-15.3)$ & 0.38 \\
\hline Midpoint between ventral part and apex & $4.3(1.5-9.0)$ & $7.4(2.5-17.1)$ & 0.10 \\
\hline \multicolumn{4}{|l|}{ Left } \\
\hline Apex & $2.56(0.7 \mathrm{I}-4.57)$ & $3.41(0.9-6.8)$ & 0.24 \\
\hline CPA & $17.4(7.8-28.2)$ & $19.9(\mid 2.3-34.3)$ & 0.38 \\
\hline Top of the diaphragm & I2.3 (8.4-25.9) & $15.4(10.7-33.9)$ & 0.11 \\
\hline Ventral part & $4.9(3.4-10.2)$ & I3.3 (4.8-20.8) & $<0.01$ \\
\hline Midpoint between apex and CPA & $9.5(3.1-16.7)$ & $14.8(5.4-16.3)$ & 0.10 \\
\hline Midpoint between ventral part and apex & $5.2(2.4-10.7)$ & $7.1(3.7-16.1)$ & 0.10 \\
\hline
\end{tabular}

Note: Data presented as median and IQR.

than in asthma patients, especially in the ventral part of the lung (Figure 6) (Supplementary videos 1, 2, $\underline{3}$, and $\underline{4}$ ).

\section{Discussion}

To the best of our knowledge, this is the first report to demonstrate decreased local lung movement during tidal breathing in COPD patients compared to asthma patients. Local lung movement during tidal breathing was significantly smaller in COPD patients than in asthma patients, especially in the ventral lung. Four-dimensional dynamicventilation $\mathrm{CT}$ scan can elucidate the local lung movement during tidal breathing. With this new technique, we can visualize "actual breathing".

We believe this impaired movement can be attributed to lung hyperinflation, loss of pulmonary elastic recoil, and increased airway resistance precipitated by smoking. It was reported that normal deformation of the lung would appear at lung areas with large respiratory movements, 
Table 7 Velocity During Inhalation of COPD Patients with Mild Emphysema and Patients with Asthma

\begin{tabular}{|l|l|l|l|}
\hline & COPD with Mild Emphysema (n=20) & Asthma (n=I I) & pvalue \\
\hline Right & & $10.7(1.6-24.7)$ \\
Apex & $7.3(0.4-13.1)$ & $79.9(48.7-116.3)$ \\
CPA & $63.4(32.4-112.1)$ & $55.9(35.6-105.0)$ & 0.09 \\
Top of the diaphragm & $42.3(20.9-84.4)$ & $48.7(19.0-74.4)$ \\
Ventral part & $20.8(1.5-13.4)$ & $35.6(11.9-70.5)$ \\
Midpoint between apex and CPA & $31.5(8.8-61.9)$ & $29.2(10.4-46.3)$ \\
Midpoint between ventral part and apex & $16.0(5.12-40.6)$ & & 0.16 \\
\hline Left & & $12.0(2.5-20.5)$ \\
Apex & $10.0(3.6-16.0)$ & $70.9(33.0-97.5)$ \\
CPA & $60.5(31.8-85.2)$ & $61.7(34.9-101.0)$ \\
Top of the diaphragm & $44.3(27.8-86.0)$ & $47.7(20.2-69.2)$ \\
Ventral part & $17.6(8.7-55.9)$ & $42.9(15.2-56.5)$ \\
Midpoint between apex and CPA & $32.6(13.2-58.9)$ & $31.8(13.0-53.0)$ \\
Midpoint between ventral part and apex & $16.7(8.5-39.5)$ & 0.03 \\
\hline
\end{tabular}

Note: Data presented as median and IQR.

Table 8 Velocity During Exhalation of COPD Patients with Mild Emphysema and Patients with Asthma

\begin{tabular}{|c|c|c|c|}
\hline & COPD with Mild Emphysema $(n=20)$ & Asthma $(n=I I)$ & $p$-value \\
\hline \multicolumn{4}{|l|}{ Right } \\
\hline Apex & $6.6(1.8-14.3)$ & $8.2(2.6-21.8)$ & 0.56 \\
\hline CPA & $72.0(35.3-14 \mid .0)$ & $83.6(44.1-123.6)$ & 0.70 \\
\hline Top of the diaphragm & $40.5(24.3-91.5)$ & $66.2(36.2-89.7)$ & 0.02 \\
\hline Ventral part & $14.9(5.6-50.9)$ & $4 I . I(I I .3-80.2)$ & $<0.01$ \\
\hline Midpoint between apex and CPA & $31.2(6.9-67.4)$ & $38.4(14.5-78.8)$ & 0.61 \\
\hline Midpoint between ventral part and apex & $15.4(5.2-38.2)$ & $24.0(7.5-60.8)$ & 0.13 \\
\hline \multicolumn{4}{|l|}{ Left } \\
\hline Apex & $8.6(3.5-16.7)$ & $10.2(3.2-21.4)$ & 0.86 \\
\hline CPA & $72.4(33.7-112.4)$ & $62.4(36.5-99.7)$ & 0.76 \\
\hline Top of the diaphragm & $50.6(25.9-85.8)$ & $49.8(32.1-95.5)$ & 0.95 \\
\hline Ventral part & $17.8(8.9-34.3)$ & $40.0(13.5-67.2)$ & $<0.01$ \\
\hline Midpoint between apex and CPA & $36.2(|I .1-5| .3)$ & $4 I .1(19.1-57.0)$ & 0.61 \\
\hline Midpoint between ventral part and apex & I $5.4(7.4-33.7)$ & $18.5(13.2-47.5)$ & 0.08 \\
\hline
\end{tabular}

Note: Data presented as median and IQR.

such as the lung base and the dorsal part of the lung, and abnormal deformation is observed throughout the lung caused by heterogeneous ventilation or partial airtrapping due to emphysema and airway disease of COPD. ${ }^{9}$ These observations suggest that abnormal pulmonary deformation by ventilation can be observed in COPD patients who smoked heavily and this differentiates them from asthma patients. Hida et al reported the maximal diaphragmatic motion in severe COPD patients during forced breathing was smaller compared to normal subjects. ${ }^{10}$ This deterioration of diaphragmatic motion indicates a limitation of diaphragm motility in COPD patients. However, they acknowledged that since they used time-resolved quantitative analysis of the diaphragms with dynamic chest radiography in the standing position and posteroanterior views only, they could not specify where the movement was diminished. We evaluated whole lung movement with four-dimensional CT scan, especially in sagittal plane. With this stereoscopic approach, we confirmed movement of the ventral part of the lung was diminished, especially during breathing, which may be useful when they undergo rehabilitation.

In addition, all patients underwent $\mathrm{CT}$ scan in the supine position. In this position, the ventral part is less 

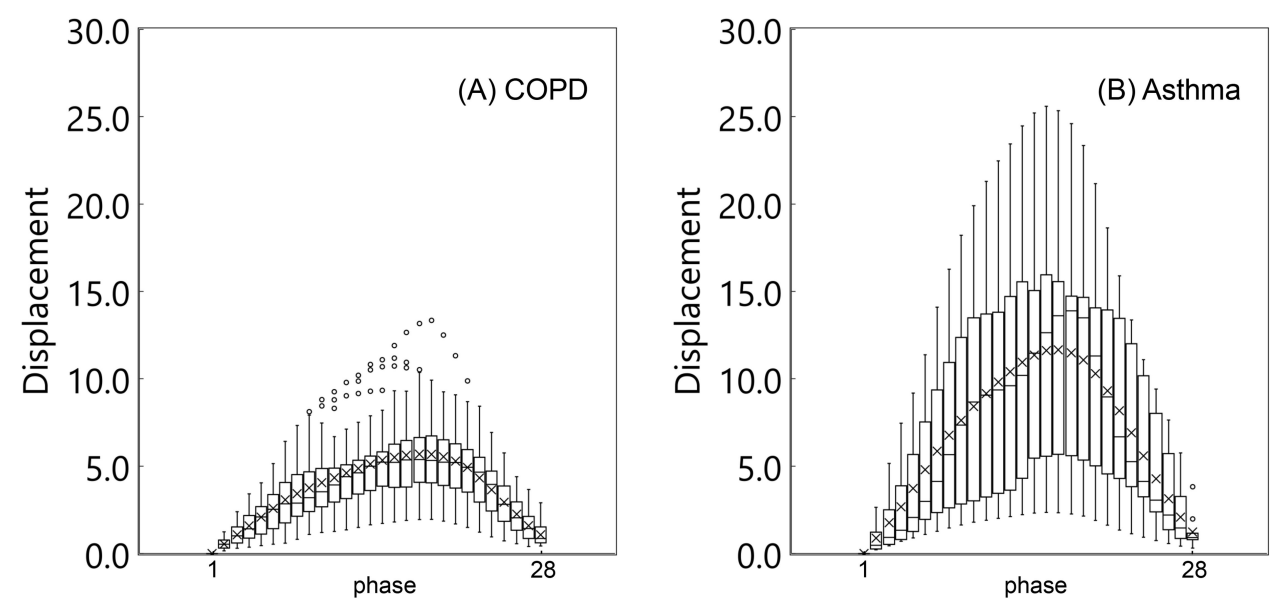

Figure 2 A series of box-plots showing displacement of the ventral point of the right lung. (A) A box-plot of COPD. (B) A box-plot of asthma. It demonstrates greater alteration for displacement in asthma patients, compared with COPD patients. Box plots of measurement of displacement and velocity value in abdominal part of the lung. "Displacement" means the amount of movement of the interest target voxel which move from the initial phase. "Velocity" means the amount of velocity of the interest target voxel which move from the previous phase. $X$ axis is the one respiratory phase starting from the level of peak inspiratory phase. The $X$-axis represents one respiratory cycle, and this is a reconstruction of one breath into 28 phases bin starting from the peak inspiratory phase. The Y-axis represents "displacement" and "velocity", and is a measurement at each respiratory phase for each patient group.

Notes: Boxes: upper to lower quartile, thin line: maximum and minimum (excluding outliers and extreme values).
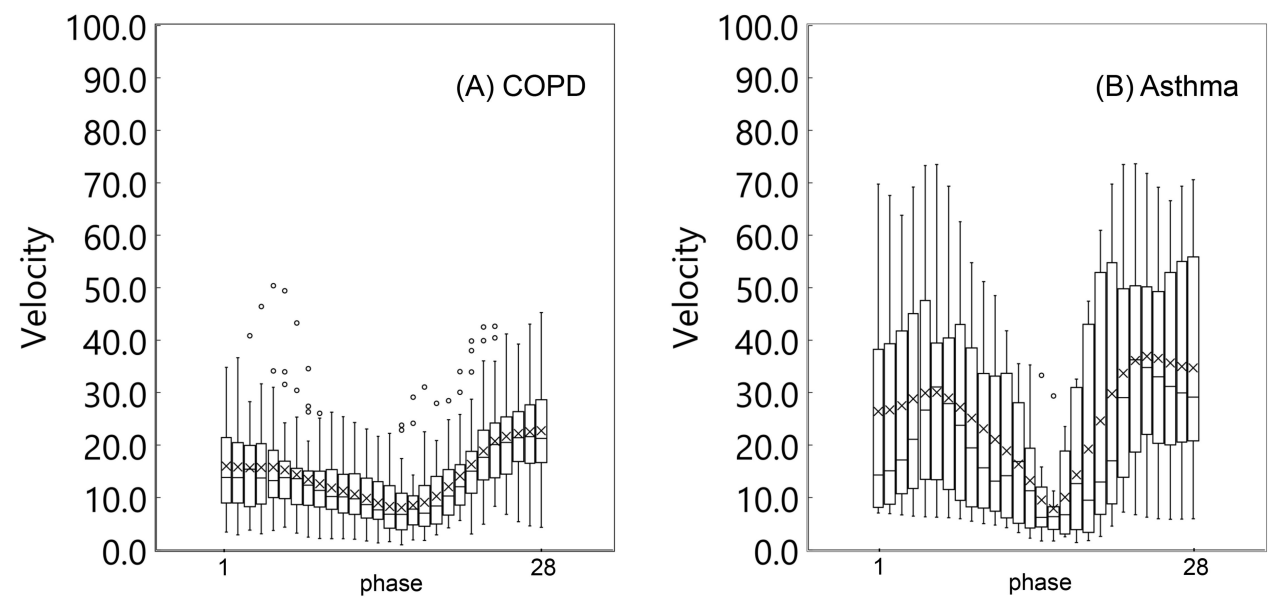

Figure 3 A series of box-plots showing velocity of the ventral point of the right lung. (A) A box-plot of COPD. (B) A box-plot of asthma. It demonstrates greater alteration for velocity in asthma patients, compared with COPD patients.

likely to be affected by gravity. Although the structure of the lung in asthma patients is fundamentally maintained, the lung structure in COPD is patients is frequently destroyed and their elastic recoil is lost by smoking. ${ }^{11}$ It is feasible that the part, less affected by gravity, tend to move less when the patient breathes. Yamashiro et al demonstrated asynchronized, heterogeneous lung movement in COPD patients. ${ }^{12}$ We believe these asynchronized and heterogeneous movements are particularly important to distinguish COPD from asthma. This abnormal movement (inability to inflate fully during inhalation) is more likely to be observed in the ventral part of the lung, the most unaffected part when the patient is in the prone position. As it is sometimes difficult to distinguish COPD from asthma, this finding may be useful.

Furthermore, we found that this decreased lung movement is already observed in COPD patients with early stage emphysema (Goddard score $<8$ ), suggesting this abnormality may develop before emphysema is radiologically evident. McDonough et al showed that the narrowing and loss of terminal bronchioles preceded emphysematous destruction in COPD and this result was confirmed in patients with mild COPD compared with controls. ${ }^{13}$ The decreased lung movement with mild emphysema in our study may be attributed to loss of terminal bronchioles and supports their findings. Further, if deterioration of lung movement can develop 

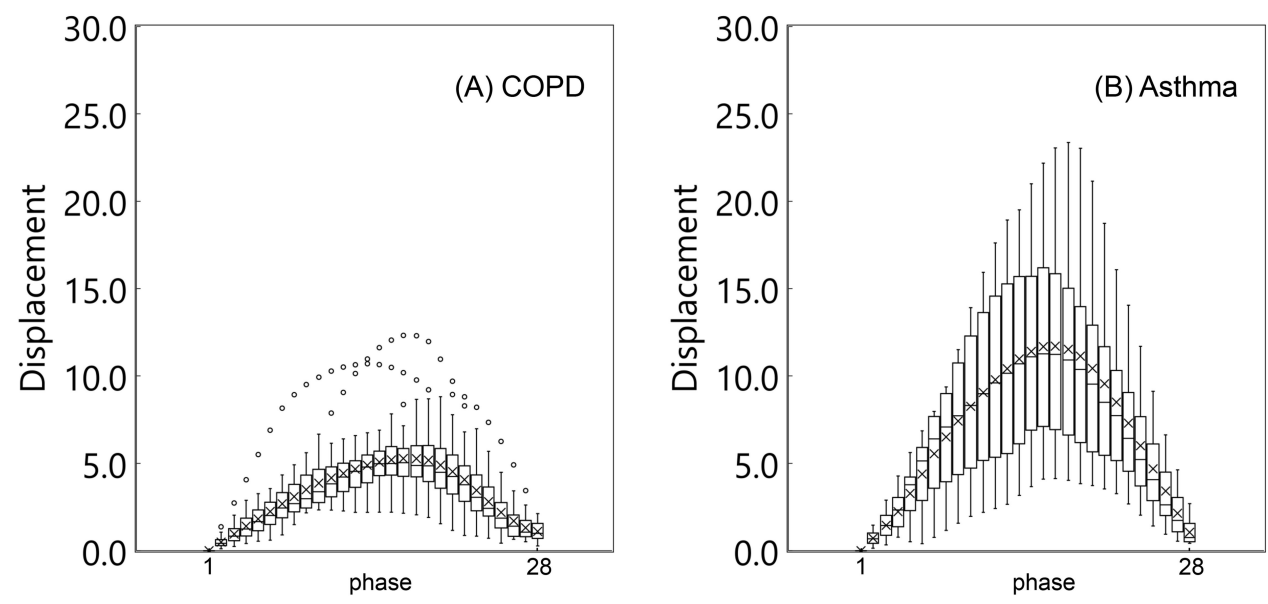

Figure $4 \mathrm{~A}$ series of box-plots showing displacement of the ventral point of the left lung. (A) A box-plot of COPD. (B) A box-plot of asthma. It also demonstrates greater alteration for displacement in asthma patients, compared with COPD patients.
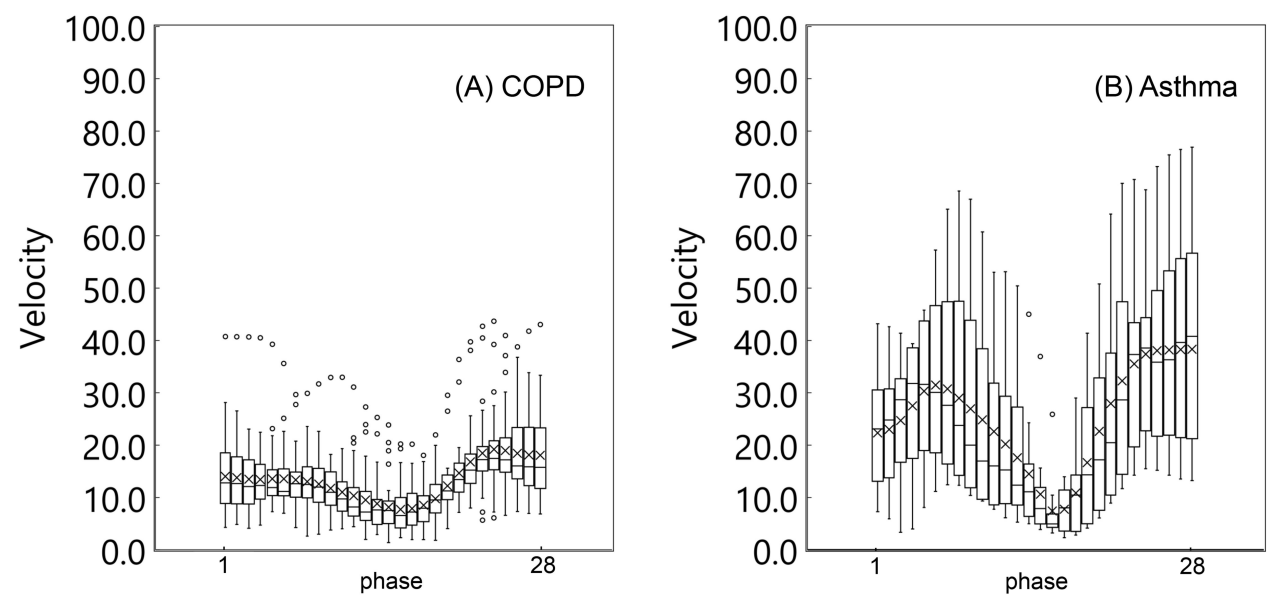

Figure 5 A series of box-plots showing velocity of the ventral point of the left lung. (A) A box-plot of COPD. (B) A box-plot of asthma. It also demonstrates greater alteration for velocity in asthma patients, compared with COPD patients.

before visible emphysematous lesions, this could be useful for COPD diagnosis at an early stage.

It should also be noted that with the available software and workstation, the four-dimensional dynamic-ventilation CT scan, used in this study, does not require any special technique. The maneuver is easy and harmless to the patients. Though patients are exposed to a slightly elevated level of radiation compared to conventional CT scan, we can obtain more information regarding early COPD diagnosis.

This study had some limitations. First, this study included 33 COPD patients and 11 asthma patients recruited at a single institution. Additional studies with a larger cohort are needed to confirm our observations. We anticipate the present study provides preliminary findings that can be validated in a larger cohort. Second, we did not evaluate how the patients were breathing, ie inter-costal or abdominal breathing. The method of breathing might have an impact on the result. Third, since there were no patients with GOLD IV, our findings may not be representative of severely ill patients. But we believe it is important that even in nonseverely ill patients, the local deterioration of lung movement can be observed. Fourth, position and phase misalignment may occur since our technique of 4DCT acquisition was retrospectively reconstructed using whole lung images and the external gating system. Fifth, there was a three-month interval between CT scan and spirometry because this was a retrospective study. Ideally, we should perform CT scan and spirometry on the same day, but as no exacerbation was observed during this period, we believe that the assessment was accurate. Sixth, our study shows a maximum estimated radiation exposure dose of $18.3 \mathrm{mSv}$, which is about three times 

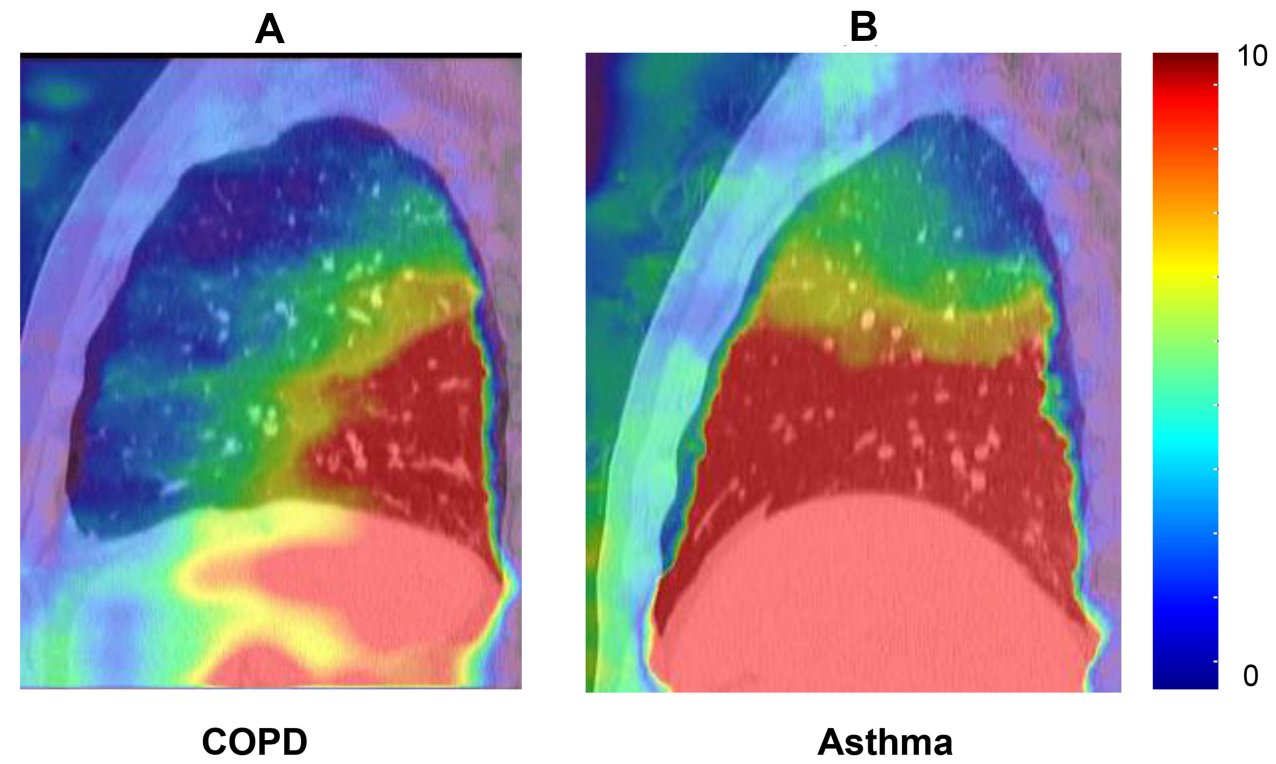

Figure 6 Typical movement of the lung. The amount of local lung movement was quantified and displayed as "displacement map". The local lung movement in COPD patients were significantly smaller than those in asthma patients especially in the ventral part of the lung. Color wash range: displacement: 0-10. (A) A typical movement of COPD. (B) A typical movement of asthma.

higher than reported by Yamashiro et al. ${ }^{12}$ However, the scan range was longer than in this previous study. Though, Yamashiro et al evaluated $16 \mathrm{~cm}$ lung span, which serves about half of the thorax, our study evaluated about twice as much as to evaluate the whole thorax. Phyziodynamics is a technology that enables seamless interpolation during motion, which enables very smooth image reconstruction. If the main purpose is not the evaluation of the lung field but the quantitative evaluation of the movement in a region with an arbitrary size, the dose might be reduced.

Furthermore, in recent years, research on CT reconstruction techniques has been actively conducted for the purpose of obtaining a low dose and high-quality image. For example, FIRST (forward projected model-based iterative reconstruction solution), which is an iterative reconstruction technology, ${ }^{14}$ and AiCE (advanced intelligent clear-IQ engine), which is an image reconstruction method using deep learning, and one of the AI technologies. ${ }^{15,16}$ Further reductions by these technologies will therefore promote reduced exposure.

In this study, the dose increase ratio was up to twofold compared to conventional static CT, but it appears useful to distinguish COPD from asthma. If radiation exposure can be reduced, our approach may be performed more readily.

\section{Conclusions}

Using four-dimensional dynamic-ventilation CT scan, we report that movement of the ventral part of the lung during tidal breathing is smaller in COPD patients than in asthma patients. This finding can be observed even when emphysema is not severe. These findings may be useful to differentiate COPD from asthma.

\section{Abbreviations}

CT, computed tomography; MDCT, multidetector computed tomography; VOI, volume of interest; CPA, costophrenic angle; LAV, low attenuation volume; GOLD, global initiative for chronic obstructive lung disease; AI, artificial intelligence.

\section{Ethics and Consent Statement}

The study protocol, any amendments, the informed consent form, and any other materials requiring preapproval were reviewed and approved by the ethics committee at Fujieda Municipal General Hospital, which was conducted in accordance with the Declaration of Helsinki. All patients provided written informed consent to participate in the study. Patient consent is not required for publication of the results as all data are anonymized.

\section{Acknowledgments}

This research did not receive any specific grant from funding agencies in the public, commercial, or not-forprofit sectors. 


\section{Author Contributions}

All authors contributed to study conception and design, acquisition of data or data analysis and interpretation; drafting and revising the article; they have agreed on the journal to which the article will be submitted, gave final approval of the version to be published; and agreed to be accountable for all aspects of the work.

\section{Funding}

There is no funding to report.

\section{Disclosure}

No funding bodies were involved in the study's design, data collection and analysis, the decision to publish, or in the preparation of the manuscript. The authors report no conflicts of interest in this work.

\section{References}

1. Abramson MJ, Perret JL, Dharmage SC, et al. Distinguishing adult-onset asthma from COPD: a review and a new approach. Int J Chron Obstruct Pulmon Dis. 2014;9:945-962.

2. Suga K, Tsukuda T, Awaya H, et al. Impaired respiratory mechanics in pulmonary emphysema: evaluation with dynamic breathing MRI. $J$ Magn Reson Imaging. 1999;10(4):510-520. doi:10.1002/(SICI) 1522-2586(199910)10:4<510::AID-JMRI3>3.0.CO;2-G

3. Simon G, Bonnell J, Kazantzis G, Waller RE. Some radiological observations on the range of movement of the diaphragm. Clin Radiol. 1969;20(2):231-233. doi:10.1016/S0009-9260(69)80181-9

4. Unal O, Arslan H, Uzun K, Ozbay B, Sakarya ME. Evaluation of diaphragmatic movement with MR fluoroscopy in chronic obstructive pulmonary disease. Clin Imaging. 2000;24(6):347-350. doi:10.1016/ S0899-7071(00)00245-X

5. Yamashiro T, Moriya H, Tsubakimoto M, et al. Continuous quantitative measurement of the proximal airway dimensions and lung density on four-dimensional dynamic-ventilation CT in smokers. Int J Chron Obstruct Pulmon Dis. 2016;11:755-764. doi:10.2147/COPD.S100658
6. Vestbo J, Hurd SS, Agusti AG, et al. Global strategy for the diagnosis, management, and prevention of chronic obstructive pulmonary disease: GOLD executive summary. Am J Respir Crit Care Med. 2013;187(4):347-365. doi:10.1164/rccm.201204-0596PP

7. GINA [homepage on the Internet]. 2020 GINA report, global strategy for asthma management and prevention. Available from: https:// ginasthma.org/gina-reports/\#: :text=2020\%20GINA\%20Report\%2C $\% 20$ Global $\% 20$ Strategy, on $\% 20$ the $\% 20$ GINA $\% 20$ Science $\%$ 20Committee. Accessed July 1, 2020.

8. Kanda Y. Investigation of the freely available easy-to-use software 'EZR' for medical statistics. Bone Marrow Transplant. 2013;48 (3):452-458. doi:10.1038/bmt.2012.244

9. $\mathrm{Xu} \mathrm{Y}$, Yamashiro T, Moriya $\mathrm{H}$, et al. Strain measurement on four-dimensional dynamic-ventilation CT: quantitative analysis of abnormal respiratory deformation of the lung in COPD. Int J Chron Obstruct Pulmon Dis. 2018;14:65-72.

10. Hida T, Yamada Y, Ueyama Y, et al. Decreased and slower diaphragmatic motion during forced breathing in severe COPD patients: time-resolved quantitative analysis using dynamic chest radiography with a flat panel detector system. Eur J Radiol. 2019;112:28-36. doi:10.1016/j.ejrad.2018.12.023

11. Aoshiba K, Nagai A. Differences in airway remodeling between asthma and chronic obstructive pulmonary disease. Clin Rev Allergy Immunol. 2004;27(1):035-044. doi:10.1385/CRIAI:27:1:035

12. Yamashiro T, Moriya H, Matsuoka S, et al. Asynchrony in respiratory movements between the pulmonary lobes in patients with COPD: continuous measurement of lung density by 4-dimensional dynamic-ventilation CT. Int J Chron Obstruct. 2017;12:2101.

13. McDonough JE, Yuan R, Suzuki M, et al. Small-airway obstruction and emphysema in chronic obstructive pulmonary disease. $N$ Engl J Med. 2011;365(17):1567-1575. doi:10.1056/NEJMoa1106955

14. Hassani C, Ronco A, Prosper AE, et al. Forward-projected model-based iterative reconstruction in screening low-dose chest CT: comparison with adaptive iterative dose reduction 3D. AJR Am $J$ Roentgenol. 2018;211(3):548-556. doi:10.2214/AJR.17.19245

15. Singh R, Digumarthy SR, Muse VV, et al. Image quality and lesion detection on deep learning reconstruction and iterative reconstruction of submillisievert chest and abdominal CT. AJR Am J Roentgenol. 2020;214(3):566-573. doi:10.2214/AJR.19.21809

16. Nakamura Y, Higaki T, Tatsugami F, et al. Possibility of deep learning in medical imaging focusing improvement of computed tomography image quality. J Comput Assist Tomogr. 2020;44(2):161-167. doi:10.1097/RCT.0000000000000928

\section{Publish your work in this journal}

The International Journal of COPD is an international, peer-reviewed journal of therapeutics and pharmacology focusing on concise rapid reporting of clinical studies and reviews in COPD. Special focus is given to the pathophysiological processes underlying the disease, intervention programs, patient focused education, and self management protocols. This journal is indexed on PubMed Central, MedLine and CAS. The manuscript management system is completely online and includes a very quick and fair peer-review system, which is all easy to use. Visit http://www.dovepress.com/testimonials.php to read real quotes from published authors. 